\title{
Antimicrobial resistance and over the counter use of drugs in Nepal
}

\author{
Sunil Pokharel ${ }^{1}$, Bipin Adhikari ${ }^{1,2}$
}

${ }^{1}$ Centre for Tropical Medicine and Global Health, Nuffield Department of Medicine, University of Oxford, Oxford, UK

${ }^{2}$ Nepal Community Health and Development Centre, Balaju, Kathmandu, Nepal

$\Lambda$ ntimicrobial resistance (AMR) is an emerging global threat. Antimicrobial resistant infections cause 700000 deaths every year globally and is estimated to account for 10 million deaths each year by 2050 if no actions taken [1]. World Health Organization has warned that the current trend in the emergence and spread of AMR could lead to 'post-antibiotic era' catastrophic consequences [2]. In Asia alone, more than 4 million deaths are projected to be due to AMR by 205 [3].

\section{BURDEN OF AMR IN SOUTH ASIA}

The risk and burden of AMR in Asia are disproportionately higher than any other continents because of high population growth triggering antimicrobial demand together with the unregulated antimicrobial use in both humans and animals, and the wide circulation of counterfeit and sub-standard medicines [4]. Asia is the most populous continent of the planet earth with the population of 4.5 billion and constitutes $60 \%$ of the world's population with China and India together constituting more than two-third of the Asia's population [5]. Together with the population density, current transition in economy, migration, high burden of infectious diseases and tropical diseases and the consequent demands and use of antimicrobials has made Asia a hub for the antimicrobial resistance [6]. The emerging resistance of antimicrobials in South Asia is intertwined with epidemiological, social, cultural and political characteristics of the nations. In this article, we will discuss the social, cultural and regulatory factors contributing to AMR and potential solutions by focusing on the context of Nepal.

Over the Counter (OTC) use of antimicrobial occurs when patients use medicines without a prescription from medical practitioner and without a medical supervision. OTC medications for common infections such as respiratory tract infections, urinary tract infections and typhoid fever by minimally qualified and unqualified allied health professionals is a common practice in developing countries where more than 50\% antimicrobials are used without a medical prescription, most commonly purchased from pharmacies or 'drug shops'. 


\section{AMR IN THE CONTEXT OF NEPAL}

In Nepal, the doctor patient ratio is still below (0.17/1000 population) the WHO recommendation (2.3/1000 population) [7]. The inequality in the distribution of health facilities and trained health human resources between urban and rural areas is alarmingly high. A huge proportion of population is deprived of the optimal health facilities creating a conducive environment for informal health care services to grow. The financial incentives to the dispensers together with poorly regulated medicine dispending practice and consumers' concern about the cost of consultation and investigations in hospital visits largely drive the over the counter (OTC)/non-prescription use of antimicrobials. The widespread myths that 'antibiotic' treat all forms of infections and are effective against viral infections undermine the role of a medical consultation [8]. Such practices of prescribing antimicrobials have been in annual rise.

In Nepal, apart from public health facilities and private hospitals/clinics, there are huge number of formal/ informal pharmacies similar to grocery shops. Drug Act 1978, and subsequent regulator frameworks have clearly mentioned antibiotics as the drug requiring prescription for dispensing. Nepal Pharmacy Council has outlined the guideline for the dispensing of prescribed drugs by a qualified and registered pharmacists [9]. However, the practice in drug-shops ubiquitous in Nepal is largely unregulated and drug dispensers have poor compliance to good pharmacy practice [10]. Hospital pharmacies located around the premises of government and private hospitals are under-equipped, under-resourced and are not readily accessible to a large population. Community pharmacies (often non-pharmacist run registered/unregistered 'drug shops') both in urban and rural Nepal generally practice irrational dispensing of the antibiotics. These unregulated 'drug shops' sell wide range of prescription only medicines which commonly include antimicrobials and often provide in higher doses exceeding the therapeutic limit and sometimes in sub-therapeutic doses [11]. In addition, in most of the cases, antimicrobials are empirically dispensed without establishing a diagnosis.

OTC use of antimicrobial occurs when patients use medicines without a prescription from medical practitioner and without a medical supervision. OTC medications for common infections such as respiratory tract infections, urinary tract infections and typhoid fever by minimally qualified and unqualified allied health professionals is a common practice in developing countries where more than $50 \%$ antimicrobials are used without a medical prescription, most commonly purchased from pharmacies or 'drug shops' [12]. A recent systematic review reported a high prevalence of self-medication with antimicrobials, most commonly amoxicillin, macrolides, fluoroquinolones, cephalosporins and metronidazole in Southeast Asia [13]. Antimicrobial use exerts antimicrobial selection pressure and contributes to the development of AMR $[14,15]$. This is more pronounced with OTC associated with inappropriate selection of antimicrobial drugs, their dose and duration of treatment [14]. Primary care facilities including the symptomatic treatment of infections were found to be the prominent reasons for high odds (range 1.4-3.7) of developing AMR [12,14].

Healthcare expenditure as a share of national GDP in Nepal has largely increased over the past decade mostly driven by out of pocket (OOP) expenditure (OOP contributed more than $60 \%$ of health care expenditure) [16]. Government expenditure on health has remained low, $11 \%$ population had health care expenditure of more than $10 \%$ of total expenses and $1.7 \%$ of total population was pushed below poverty line in 2016 due to health care expenses [16]. Public health delivery system is ineffective even in urban areas due to poor governance, lack of skilled human resources and quality laboratory services. Private health facilities are expensive (high cost associated with diagnosis, treatment, travel and opportunity cost) and out of reach to a large population. Government of Nepal has recently implemented social health insurance system which is challenged by low acceptability and coverage ( $5 \%$ in the implemented districts) and is a long way from significantly contributing to universal health coverage [17]. The huge OOP health expenditure has rendered relatively cheaper OTC a first option in health care seeking and hospital consultation a last resort.

Research to understand the trends in antimicrobial use and factors driving the OTC antimicrobial use, and follow-up address to the knowledge gaps through policy interventions is urgently required.
Apart from apparent financial shortcuts of visiting pharmacies, in Nepal and in other South Asian countries, both formal and informal Ayurvedic and traditional practitioners are attended by large proportion of the population [18]. Unregulated Ayurvedic and traditional practitioners often empirically use antimicrobials either in the form of certified allopathic product or uncertified plant extracts with antimicrobial property to treat infections. Such a practice, in fact is distracting the early health seeking behavior. Augmented by the socio-demographic barriers (distance, direct and indirect costs associated with attending hospital), traditional beliefs 


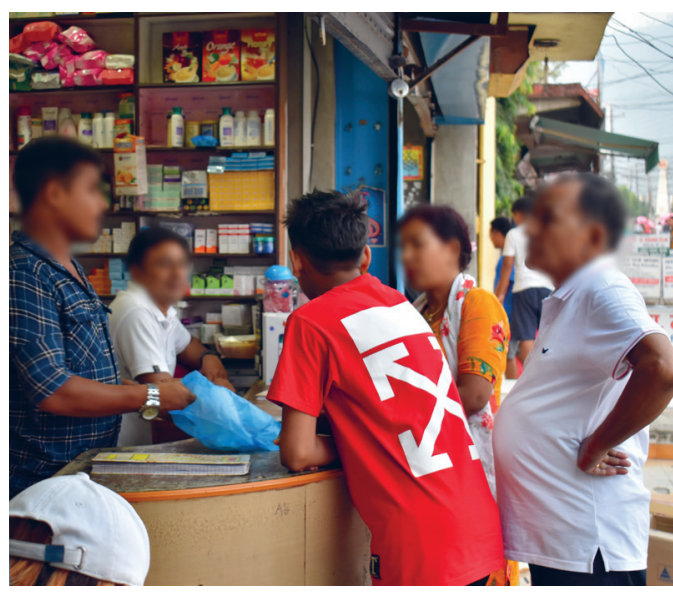

Photo: From the collection of Dr Achyut Adhikari (used with permission). and practices, patient may easily fall into a cycle of poly visits to traditional healers and medical dispensers thus propitiating the development of AMR.

\section{WAY FORWARD}

Legislation in Nepal mandates prescription for dispensing of an antimicrobial drug by a registered pharmacist. Strict enforcement of regulation, particularly in dispensing of the antibiotics by the pharmacies, together with development and adherence to national standard treatment protocol, is urgently required. Universal health care with increased access to public health facilities and health insurance for financial protection against catastrophic private health care expenditure is critical. Nevertheless, these interventions alone are insufficient to stop OTC dispensing of antimicrobials in the socio-economic context of Nepal and will be futile without addressing the social, cultural and behavioral factors driving the OTC antimicrobial use. Research to understand the trends in antimicrobial use and factors driving the OTC antimicrobial use, and follow-up address to the knowledge gaps through policy interventions is urgently required.

Disclaimer: All authors contributed in their individual capacities and the views that are expressed in this commentary do not necessarily reflect the views of their respective organization.

Funding: None.

Authorship contributions: SP and BA conceived and drafted the manuscript including revisions for this commentary. The authors read and approved the final manuscript.

Competing interests: The authors have completed the ICMJE Unified Competing Interest form (available on request from the corresponding author) and declare no competing interests.

1 O'Neill J. Tackling Drug-Resistance Infections Globally: Final Report and Recommendations. 2016. Available: https:// amr-review.org/sites/default/files/160518_Final\%20paper_with\%20cover.pdf. Accessed: 8 June 2019.

2 World Health Organization. Global Antimicrobial Resistance Surveillance System (GLASS) Report: Early implementaion 2016-17. Available: https://apps.who.int/iris/bitstream/handle/10665/259744/9789241513449-eng.pdf;jsessionid=27C40B6227E08D2B0BE7EB12C7E21BE4?sequence=1. Accessed: 8 June 2019.

3 O'Neill J. AMR Review Paper-Tackling a crisis for the health and wealth of nations. 2014. Available: https://amr-review. org/sites/default/files/AMR\%20Review\%20Paper\%20-\%20Tackling\%20a\%20crisis\%20for\%20the\%20health\%20 and\%20wealth\%20of\%20nations_1.pdf. Accessed: 8 June 2019.

4 Pokharel S, Raut S, Adhikari B. Tackling antimicrobial resistance in low-income and middle-income countries. BMJ Glob Health. 2019;4:e02104. Medline:31799007 doi:10.1136/bmjgh-2019-002104

5 Worldometers. Current World Population 2019. 2019. Available: https://www.worldometers.info/world-population/. Accessed: 10 December 2019.

6 Horby PW, Pfeifer D, Oshitani H.. Prospects for emerging infections in East and Southeast Asia 10 years after severe acute respiratory syndrome. Emerg Infect Dis. 2013;19:853. Medline:23738977 doi:10.3201/eid1906.121783

7 Adhikari B, Mishra SR. Urgent need for reform in Nepal's medical education. Lancet. 2016;388:2739-40. Medline:27924772 doi:10.1016/S0140-6736(16)32423-0

8 World Health Organization. Worldwide country situation analysis: response to antimicrobial resistance. Geneva: WHO; 2015.

9 Nepal Pharmacy Council. National Good Pharmacy Practice Guidelines. Kathmandu: Nepal Pharmacy Council; 2005.

10 Shrestha R, Ghale A. Study of good pharmacy practice in community pharmacy of three districts of Kathmandu valley, Nepal. Int J Sci Rep. 2018;4:240-5. doi:10.18203/issn.2454-2156.IntJSciRep20184191

11 Miller R, Goodman C. Performance of retail pharmacies in low- and middle-income Asian settings: a systematic review. Health Policy Plan. 2016;31:940-53. Medline:26962123 doi:10.1093/heapol/czw007

12 Morgan DJ, Okeke IN, Laxminarayan R, Perencevich EN, Weisenberg S. Non-prescription antimicrobial use worldwide: a systematic review. Lancet Infect Dis. 2011;11:692-701. Medline:21659004 doi:10.1016/S1473-3099(11)70054-8

13 Nepal G, Bhatta S. Self-medication with Antibiotics in WHO Southeast Asian Region: A Systematic Review. Cureus. 2018;10:e2428. Medline:29876150 doi:10.7759/cureus.2428 
14 Costelloe C, Metcalfe C, Lovering A, Mant D, Hay AD. Effect of antibiotic prescribing in primary care on antimicrobial resistance in individual patients: systematic review and meta-analysis. BMJ. 2010;340:c2096. Medline:20483949 doi:10.1136/bmj.c2096

15 Bell BG, Schellevis F, Stobberingh E, Goossens H, Pringle M. A systematic review and meta-analysis of the effects of antibiotic consumption on antibiotic resistance. BMC Infect Dis. 2014;14:13. Medline:24405683 doi:10.1186/1471-233414-13

16 World Health Organization. Health financing profile 2017. Available: https://apps.who.int/iris/bitstream/handle/10665/259643/HFP-NEP.pdf?sequence=1. Accessed: 8 June 2019.

17 Pokharel R, Silwal PR. Social health insurance in Nepal: A health system departure toward the universal health coverage. Int J Health Plann Manage. 2018. Epub ahead of print. Medline:29635799 doi:10.1002/hpm.2530

18 Adhikari B, Mishra RS. Culture and epidemiology of diabetes in South Asia. J Glob Health. 2019;9:020301. Medline:31448112 doi:10.7189/jogh.09.020301

\section{Correspondence to:}

Dr. Bipin Adhikari

Centre for Tropical Medicine and Global Health

Nuffield Department of Medicine

Peter Medawar Building

University of Oxford

Oxford, OX1 3SY

UK

biopion@gmail.com 